\title{
Absolute calibration of brightness temperature of the Venus disk observed by the Longwave Infrared Camera onboard Akatsuki
}

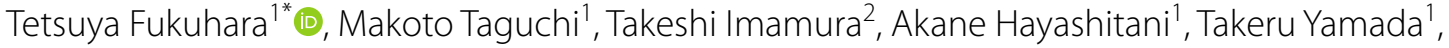 \\ Masahiko Futaguchi ${ }^{3}$, Toru Kouyama ${ }^{4}$, Takao M. Sato ${ }^{5}$, Mao Takamura ${ }^{1}$, Naomoto Iwagami ${ }^{6}$, Masato Nakamura ${ }^{5}$, \\ Makoto Suzuki ${ }^{5}$, Munetaka Ueno ${ }^{7}$, George L. Hashimoto ${ }^{8}$, Mitsuteru Sato ${ }^{9}$, Seiko Takagi ${ }^{10}$, Atsushi Yamazaki ${ }^{5}$, \\ Manabu Yamada ${ }^{11}$, Shin-ya Murakami ${ }^{5}$, Yukio Yamamoto ${ }^{5}$, Kazunori Ogohara ${ }^{12}$, Hiroki Ando ${ }^{13}$, \\ Ko-ichiro Sugiyama ${ }^{14}$, Hiroki Kashimura', Shoko Ohtsuki ${ }^{6}$, Nobuaki Ishii ${ }^{5}$, Takumi Abe ${ }^{5}$, Takehiko Satoh ${ }^{5}$, \\ Chikako Hirose $^{5}$ and Naru Hirata ${ }^{15}$
}

\begin{abstract}
The Venus Climate Orbiter Akatsuki arrived at Venus in December 2015, and the Longwave Infrared Camera (LIR) onboard the spacecraft started making observations. LIR has acquired more than 8000 images during the first two Venusian years since orbit insertion without any serious faults. However, brightness temperature derived from LIR images contained an unexpected bias that related not to natural phenomena but to a thermal condition of the instrument. The bias could be partially eliminated by keeping the power supply unit for LIR always active, while the residual bias was simply correlated with the baffle temperature. Therefore, deep-space images were acquired at different baffle temperatures on orbit, and a reference table for eliminating the bias from images was prepared. In the corrected images, the brightness temperature was $\sim 230 \mathrm{~K}$ at the center of the Venus disk, where the effect of limb darkening is negligible. The result is independent of the baffle temperature and consistent with the results of previous studies. Later, a laboratory experiment with the proto model of LIR showed that when the germanium (Ge) lens was heated, its actual temperature was slightly higher than the temperature measured by a thermal sensor attached to the lens holder. The experiment confirmed that transitory baffle heating accounted for the background bias found in the brightness temperature observed by LIR.
\end{abstract}

Keywords: Venus, Thermal infrared, Akatsuki

\section{Introduction}

The Venus Climate Orbiter Akatsuki was successfully inserted into a Venus orbit in December 2015 after spending 5 years orbiting the sun under unintended environmental conditions. Subsequently, five cameras and an ultra-stable oscillator for a radio occultation experiment onboard Akatsuki started observation of the Venus atmosphere (Nakamura et al. 2016). The Longwave

\footnotetext{
*Correspondence: tetsuyaf@rikkyo.ac.jp

${ }^{1}$ College of Science, Rikkyo University, 3-34-1 Nishi-Ikebukuro,

Toshima-ku, Tokyo 171-8501, Japan

Full list of author information is available at the end of the article
}

Infrared Camera (LIR), which is one of the five cameras onboard Akatsuki, maps of brightness temperature of the Venus disk on both day and night hemispheres by detecting emissions at wavelengths from 8 to $12 \mu \mathrm{m}$ (Fukuhara et al. 2011). Radiative transfer calculations using a typical cloud height distribution of Venus atmosphere indicate that the thermal infrared radiation emitted from an altitude of $\sim 65 \mathrm{~km}$ contributes most to the thermal contrast seen in the LIR images (Taguchi et al. 2007). The brightness temperature at that altitude is generally $\sim 230 \mathrm{~K}$ according to the vertical temperature distribution of the Venus atmosphere derived from previous observations (e.g., Seiff et al. 1985; Pollack et al. 1993; Zasova et al. 
2007; Tellman 2009). Thus, the wavelength band of LIR was designed to observe the temperatures expected at $\sim 65 \mathrm{~km}$. The noise equivalent temperature difference (NETD), which describes relative temperature resolution, is $\sim 0.3 \mathrm{~K}$ when the temperature of a target is $\sim 230 \mathrm{~K}$. The NETD corresponds to an altitude difference of a few hundred meters at the cloud-top layer on Venus (Fukuhara et al. 2011).

Immediately after the orbit insertion of the spacecraft, LIR discovered a large stationary gravity wave that appeared above Aphrodite terra close to 18:00 local solar time (Fukuhara et al. 2017). Although LIR cannot directly observe this wave at lower altitudes, a simulation showed that a wave generated by an atmospheric perturbation caused by the surface topography could propagate to the cloud-top layer. Since 1- and 2- $\mu \mathrm{m}$ cameras (IR1 and IR2) observe an aspect of the lower atmosphere in nightside by detecting the wavelengths in the atmospheric windows of Venus (Iwagami et al. 2011; Satoh et al. 2015), comparison of images acquired by LIR with those acquired by IR1 and IR2 should reveal the propagation process of the stationary gravity wave in some detail.

The radio occultation experiment termed Radio Science (RS) retrieves vertical profiles of the atmospheric temperature, the pressure, mixing ratio of the sulfuric acid vapor, and the electron density (Imamura et al. 2011). Synchronous observation by LIR and RS identifies the altitude of the cloud-top layer observed by LIR. On the other hand, Ultraviolet Imager (UVI), which is sensitive to wavelengths of 283 and $365 \mathrm{~nm}$, observes $\mathrm{SO}_{2}$ and an unknown UV absorber distributed above $\sim 65 \mathrm{~km}$ (Nakamura et al. 2011). Observations by UVI and LIR are typically synchronized. When horizontal distributions of the UV absorbers derived from UVI observation are correlated with the brightness temperature distributions derived from LIR observation, the altitude of the UV absorbers can be identified by comparison of the UV images with LIR images. Furthermore, vertical variation of the UV absorbers may be revealed by the continuously synchronized observation.

Brightness temperatures derived from a sequence of LIR images are expected to show signatures of thermal tides, convection and perhaps other atmospheric processes. Further, since LIR measures a major portion of the thermal emission from Venus, the accumulated data will provide useful information about the radiative balance of its atmosphere. The LIR images acquired immediately after the failure of orbit insertion in 2010 showed that the mean brightness temperature at the low latitudes in the nightside of Venus was $\sim 243 \mathrm{~K}$. This temperature is higher than those previously inferred and not consistent with the expected variation. The reason for the discrepancy could not be determined at this time, despite some speculation about local time dependence, as discussed in Taguchi et al. (2012).

As discussed above, not only thermal contrast but also brightness temperature is a major scientific product for LIR. Moreover, the brightness temperature is often compared with the results acquired from other instruments mounted on the spacecraft in order to retrieve physical quantities. Accuracy of the physical quantities derived from such comparisons partly depends on the accuracy of the absolute brightness temperature, whose error was estimated in the design process at $\sim 3 \mathrm{~K}$ as a standard deviation of brightness temperature at $230 \mathrm{~K}$. The standard deviation may have increased due to degradation of the sensitivity of the detector during 5 years of extended orbiting around the sun, or some other unexpected condition affecting LIR at the time of observation. The unexpected standard deviation would introduce some ambiguity in the interpretation of the atmospheric processes. For instance, the temperature discrepancy seen in Taguchi et al. (2012) is a suspicious case in which a systematic bias far larger than $3 \mathrm{~K}$ standard deviation may be involved. Therefore, we undertook an effort to verify the performance of LIR observation on orbit at an early stage so that any error could be precisely estimated.

\section{LIR instrumentation}

\section{The uncooled microbolometer array}

LIR uses an uncooled microbolometer array (UMBA) as the detector with $328 \times 248$ pixels. The internal layout of the camera is shown in Fig. 1 (Fukuhara et al. 2011). The UMBA does not use a cryogenic system, which makes the camera lightweight and small. However, components of

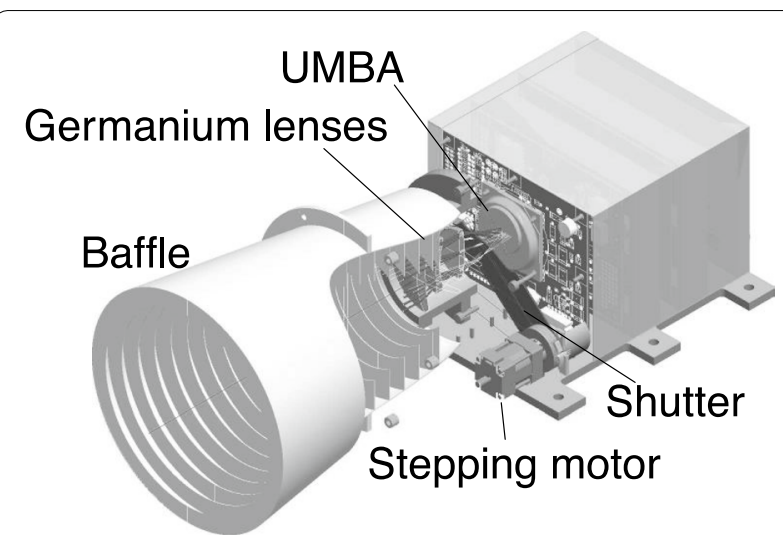

Fig. 1 Schematic of the internal layout of LIR referred from Fukuhara et al. (2011). The uncooled microbolometer camera works at room temperature. Hence, the baseplate on which the camera is mounted and the Ge lens on the camera are kept at room temperature by heaters. Temperature of the UMBA is controlled at $313 \pm 0.01 \mathrm{~K}$ by the Peltier device to keep high sensitivity. Temperature of the baffle residing outside of the spacecraft varies with the solar incident angle 
the camera still need to be thermally controlled to prevent imaging noise. Therefore, temperature of the UMBA is controlled at $313 \pm 0.01 \mathrm{~K}$ by a Peltier device. The germanium (Ge) lens on the camera and the baseplate on which the camera is mounted are kept thermally stable by heaters. The power supply unit, which is not thermally controlled, is separated from the camera so that the temperature in the vicinity of the detector does not increase. A baffle resides on the outside of the spacecraft to prevent solar radiation from directly entering the detector; however, its temperature varies drastically with the solar incident angle. Therefore, the camera is thermally isolated from the baffle.

Sensitivity of each pixel of the UMBA used in the LIR has a large inhomogeneity, which is called as on-chip fixed pattern noise (OFPN) (Fukuhara et al. 2011). The OFPN is partially subtracted from an image with the calibration data in the analog circuit of LIR. The residual component can also be eliminated from the target image by subtracting a dark image acquired by taking a shutter. Images are acquired and accumulated continuously at a rate of $60 \mathrm{~Hz}$ in order to reduce random noise; we defined the number of accumulations as " $m$." The procedure is continuously repeated up to 32 times within $120 \mathrm{~s}$, and resultant images are further accumulated; we defined the number of repetitions as " $n$." Digital electronics (DE) in the spacecraft process the first and second accumulations. Then, the averaged image is stored in a data recorder and subsequently sent to the ground station.

\section{Conversion of data counts to brightness temperature}

The original LIR image sent from the spacecraft expresses brightness detected with 12 bits per each pixel. The data number for each pixel is converted to brightness temperature based on the reference data, which were derived from the blackbody images acquired during calibration tests at Institute of Space and Astronautical Science (ISAS) of JAXA, in a vacuum environment before launch (Fukuhara et al. 2011). The blackbody used in the experiment was thermally controlled $5 \mathrm{~K}$ steps from 210 to $250 \mathrm{~K}$, which corresponds to the expected temperature range at the cloud tops of Venus (e.g., Taylor 1980). The original brightness contrast depends on the shutter temperature, which is not thermally controlled. Therefore, the heater attached to the baseplate of LIR controlled the temperature of the camera to balance the shutter temperature at 297, 300, and $303 \mathrm{~K}$ when the blackbody was set at each temperature. An offset of the brightness contrast caused by variation of the shutter temperature can be eliminated from each pixel by

$$
I_{\mathrm{s}}=I_{\mathrm{o}}+C_{\mathrm{s}}\left(T_{\mathrm{s}}-T_{0}\right),
$$

where $I_{\mathrm{s}}$ and $I_{\mathrm{o}}$ are the brightness values after and before the correction, respectively; $C_{\mathrm{s}}$ is the coefficient experimentally derived in Taguchi et al. (2012); and $T_{\mathrm{s}}$ and $T_{0}$ are the shutter temperature measured at the time of observation and a standard temperature properly defined for the correction based on the calibration experiments, respectively.

Since integration of the spectral radiance $I$ from $8 \mu \mathrm{m}$ $\left(\lambda_{1}\right)$ to $12 \mu \mathrm{m}\left(\lambda_{2}\right)$ is a linear function of $I_{\mathrm{s}}$ in Eq. (1), it can be represented by

$$
B=\int_{\lambda_{1}}^{\lambda_{2}} I \mathrm{~d} \lambda=G \times I_{\mathrm{s}}+C_{\mathrm{o}}
$$

where $G$ and $C_{o}$ are the gain and offset, respectively, derived from blackbody images obtained in the laboratory. When emissivity of the thermal infrared radiation from Venus is assumed to be unity, brightness temperature $T$ for each pixel in an image is derived from the Planck's law. On the other hand, $T$ can also be approximated by a power series of $B$ as follows in case the target temperature is from 210 to $250 \mathrm{~K}$ :

$$
T=\sum_{i=0}^{n} a_{i} B^{i},
$$

where the coefficients $a_{i}$ are estimated from the blackbody images and $n=7$. In this paper, formula (3) is used for converting the thermal contrast to the brightness temperature.

\section{Initial checkout of the instrument on orbit}

LIR has continuously acquired more than 8000 images without serious faults for the first two Venus years since the orbit insertion. The mechanical shutter with a stepping motor, whose prototype has been driven 650,000 times in the laboratory experiment (Fukuhara et al. 2011), has been operated 86,000 times on orbit without any mechanical trouble. However, the observational lifetime of Akatsuki was originally designed for 4 years after a half-year cruising (Nakamura et al. 2011). Therefore, major elements that may affect contrast in LIR images were initially checked on orbit.

\section{Sensitivity of the UMBA}

The sensitivity of the UMBA for LIR should not deteriorate noticeably for 10 years in a moderate storage environment. Furthermore, the UMBA experimentally endured a total dose of 300 Gy generated by a proton beam with $100 \mathrm{MeV}$ (Fukuhara et al. 2011). However, whether the sensitivity would degrade due to exposure to actual solar radiation for 5 years in an unexpected environment was still unknown. Since the sensitivity 
for each pixel is independent of other pixels, deviation of the OFPN should generally increase with deterioration of the sensitivity. Calibration data for reduction in the OFPN were prepared and installed to DE before the launch, whereas the calibration data can be updated after the launch. Therefore, the calibration data were temporarily renewed on orbit, and a Venus image with $m=1$ and $n=1$ was acquired. At the same time, another Venus image with $m=1$ and $n=1$ was acquired by using the original calibration data. The standard deviation of brightness temperature of the Venus disk was $6.5 \mathrm{~K}$ in the original image, while it was $5.4 \mathrm{~K}$ in the newly calibrated image. The difference will not be significant when an averaged image is taken into account in the actual observation. Thus, we have used the original calibration data for observations.

\section{Accumulation numbers for the observation}

Since the best NETD was acquired in the experiment before the launch when $m=32$ and $n=32$ were estimated, these parameters are used in the observation programs on the spacecraft (Fukuhara et al. 2011). The numbers can be modified on orbit in case the NETD becomes noticeably worse due to aging or other degradation. In order to verify whether the numbers were still suitable for observation, images of the Venus disk were sequentially acquired with $m=8,16,32,64$, and 128; then a standard deviation of brightness temperature inside of each Venus disk was normalized with that of $m=8$. Likewise, the standard deviation was derived from each image of a blackbody acquired in a vacuum environment at $230 \mathrm{~K}$ before the launch (Fukuhara et al. 2011). The standard deviations acquired on orbit were virtually the same as those acquired in the laboratory as listed in Table 1. Thus, the original number $m$ did not need to be modified.

An observation sequence with $n=32$ was prepared for the original orbit plan in which the spacecraft constantly observes Venus except near periapsis in a 31-h elliptical orbit (Nakamura et al. 2007). However, the current orbit with a period of 10 days is much more elongated than the original orbit plan (Nakamura et al. 2016). Distance from

Table 1 Standard deviations of the Venus disk and the blackbody temperatures normalized by the value of $m=8$

\begin{tabular}{lll}
\hline $\boldsymbol{m}$ & Venus disk on & Blackbody at 230 K on \\
\hline & March 16, 2016 & November 14, 2009 \\
8 & 1 & 1 \\
16 & 0.991 & 0.973 \\
32 & 0.986 & 0.988 \\
64 & 0.967 & 0.969 \\
128 & 0.965 & 0.953 \\
\hline
\end{tabular}

the spacecraft to Venus at the apoapsis, which was originally estimated at $79,000 \mathrm{~km}$, increased to $370,000 \mathrm{~km}$. Consequently, the spatial resolution of a Venus image acquired at the apoapsis on the current orbit becomes nearly five times worse than that originally expected. The Venus disk needs to be observed near periapsis so that images can be obtained at the spatial resolution originally expected. An observation sequence of $n=32$ with $120 \mathrm{~s}$ leads to blurring in the Venus disk image because of the high relative velocity of the spacecraft and Venus at periapsis. Thus, an observation program with $m=32$ and $n=1$ was additionally prepared for the periapsis observation, as listed in Table 2. Venus images have been constantly acquired from the orbit near periapsis by using this observation program since October 2016.

\section{Background variation in the observed image}

Average temperatures of the Venus disk and deep space were retrieved from the brightness temperature of each LIR image derived from formula (3). Figure 2 shows the temperature variations in the first Venusian year after the orbit insertion. Both the Venus disk and deep space have a temperature variation with amplitude of more than $20 \mathrm{~K}$ and a similar trace. Since formula (3) does not follow Plank's law except at temperatures from 210 to $250 \mathrm{~K}$, the deep-space temperature should converge at $\sim 180 \mathrm{~K}$. However, the large variation shown in Fig. 2 is contrary to this expectation. The short-term variation seen in the

\section{Table 2 Major observation programs for LIR}

\begin{tabular}{|c|c|c|c|}
\hline Observation name & $\begin{array}{l}\text { Instruments synchronously } \\
\text { observing with LIR }\end{array}$ & $m$ & $n$ \\
\hline Nightside deluxe & $\operatorname{IR} 2(1.73,2.26,2.02,2.32 \mu \mathrm{m})$ & 32 & 32 \\
\hline Nightside slim & IR2 $(2.26 \mu \mathrm{m})$ & 32 & 32 \\
\hline Dayside deluxe & $\begin{array}{l}\text { IR1 }(0.9 \mu \mathrm{m}), \text { IR2 }(2.02 \mu \mathrm{m}) \text {, } \\
\quad \text { UVI }(283,365 \mathrm{~nm})\end{array}$ & 32 & 32 \\
\hline Dayside slim & IR1 $(0.9 \mu \mathrm{m}), \mathrm{IR} 2(2.02 \mu \mathrm{m})$ & 32 & 32 \\
\hline Vicinity deluxe & UVI $(283,365 \mathrm{~nm})$ & 32 & 01 \\
\hline Vicinity slim & UVI $(283,365 \mathrm{~nm} \times 2)$ & 32 & 01 \\
\hline Vicinity slim & UVI $(283,365 \mathrm{~nm} \times 7)$ & 32 & 01 \\
\hline Nightside vicinity scan & IR2 $(1.73,2.26 \mu \mathrm{m})$ & 32 & 01 \\
\hline Dayside vicinity scan & $\begin{array}{l}\text { IR1 }(0.9 \mu \mathrm{m}), \text { IR2 }(2.02 \mu \mathrm{m}) \\
\quad \text { UVI }(365 \mathrm{~nm})\end{array}$ & 32 & 01 \\
\hline Nightside deluxe & $\begin{array}{l}\text { IR2 }(1.73,2.26,2.02,2.32 \mu \mathrm{m}) \\
\quad \text { with ROI }\end{array}$ & 32 & 01 \\
\hline Nightside slim & IR2 $(2.26 \mu \mathrm{m})$ with $\mathrm{ROI}$ & 32 & 32 \\
\hline Dayside deluxe & $\begin{array}{l}\text { IR1 }(0.9 \mu \mathrm{m}), \text { IR2 }(2.02 \mu \mathrm{m}) \\
\quad \text { UVI }(283,365 \mathrm{~nm})\end{array}$ & 32 & 32 \\
\hline Dayside slim & $\begin{array}{l}\text { IR1 }(0.9 \mu \mathrm{m}), \text { IR2 }(2.02 \mu \mathrm{m}) \\
\quad \text { UVI }(283,365 \mathrm{~nm}) \text { with ROI }\end{array}$ & 32 & 32 \\
\hline
\end{tabular}

An additional observation with $m=32$ and $n=1$ was prepared on October 2016

$R O /$ region of interest: a specific area selected from an images of IR1, IR2, and UVI in order to limit data quantity 


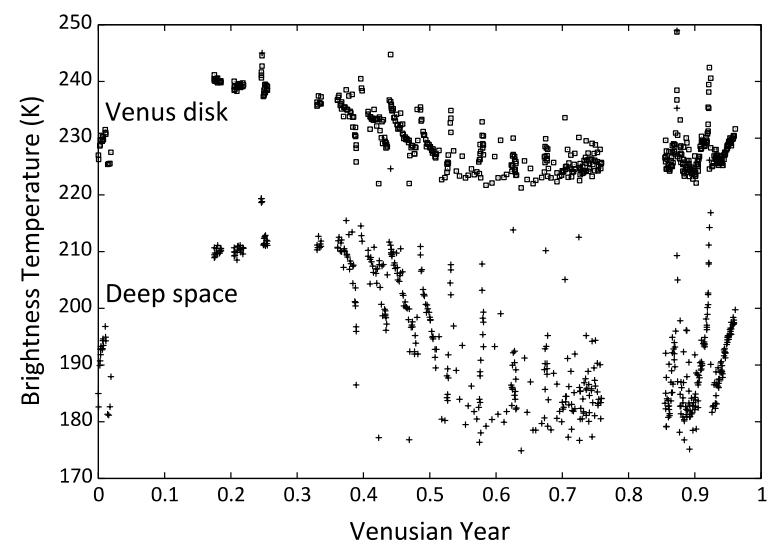

Fig. 2 Temperature variations of the Venus disk and the deep space derived from images obtained by LIR during the first Venusian year after the orbit insertion. The short-term variation that has a period of 0.045 Venusian years is coincident with the orbital period of approximately 10 earth days

figure is coincident with the orbital period of approximately 10 earth days. Meanwhile, the long-term variation depends on the orbital period of Venus, which gradually changes the geometry of the spacecraft and Venus at the time of observation. The baffle and the power supply unit were major suspects for the temperature variations because they are not thermally stabilized, and hence, they could be the source of background bias.

\section{Correction of the background bias}

Temperature stabilization of the power supply unit for LIR The brightness temperature distribution of deep space as a function of the baffle temperature is shown in Fig. 3a, where images were obtained from March to September 2016 with $m=32$ and $n=32$. The distribution is correlated with the baffle temperature, but it has obviously large deviation, especially when the baffle temperature is less than $240 \mathrm{~K}$. Originally, LIR was turned on $15 \mathrm{~min}$ before the beginning of each observation to give the instrument enough time to thermally stabilize. However, this time interval proved to be insufficient to thermally stabilize the power supply unit. Therefore, the power supply unit has been kept active since October 2016 to ensure that it is thermally stable. Consequently, the large deviation in brightness temperature (Fig. 3a) disappeared, after October 2016 (Fig. 3b). After October 2016, the deep-space temperature was simply correlated with the baffle temperature.

\section{Correction of the background bias due to baffle temperature}

Although temperatures of the baffle and the UMBA are not directly linked, the deep-space temperature in the images was correlated with the baffle temperature. Since the Ge lens in front of the UMBA is covered with the baffle, the UMBA could indirectly absorb a part of the thermal infrared radiation emitted by the baffle via the Ge lens. When the contribution ratio of the thermal radiation from the baffle is defined as $C_{\mathrm{B}}$ for each pixel, the background bias can be canceled by using the following formula from an arbitrary image in which influence of the OFPN has been eliminated by using formula (1):

$$
I_{\mathrm{c}}=I_{\mathrm{s}}-C_{B}\left(B\left(T_{\mathrm{b}}\right)-B\left(T_{\mathrm{b} 0}\right)\right),
$$

where $I_{\mathrm{c}}$ is the corrected brightness value, and $B\left(T_{\mathrm{b}}\right)$ and $B\left(T_{\mathrm{b} 0}\right)$ are the blackbody radiation emitted from the baffle at the time of observation and a standard environment, respectively. $T_{\mathrm{b} 0}$ is defined as $230 \mathrm{~K}$ based on the laboratory experiment before the launch. $C_{B}$ can be estimated from deep-space images with different baffle temperatures. Therefore, LIR acquired a deep-space image every $2 \mathrm{~h}$ with the solar incident angle defined in Fig. 4 being controlled every $15^{\circ}$ from $30^{\circ}$ to $120^{\circ}$. Furthermore, LIR was kept turned on during the observation so that temperatures of the instrument could be kept in thermal equilibrium. Thus, $C_{B}$ could be obtained from the deep-space images and $I_{c}$ for each image was derived by formula (4). $I_{\mathrm{c}}$ can be finally converted to the brightness temperature by substituting $I_{\mathrm{c}}$ instead of $I_{\mathrm{s}}$ into formula (3). Example images before and after the background correction are shown in Fig. 5.

We corrected the background bias in LIR images acquired with $m=32$ and $n=32$ from October to December 2016. Figure 6 shows a diagram in which the distribution of deep-space temperatures after the correction is added to those before the correction shown in Fig. 3b. The temperature distribution after the correction converges to $181 \mathrm{~K}$, which is independent of the baffle temperature. The standard deviation of the temperature distribution for the deep space was $3.91 \mathrm{~K}$, from which the standard deviation of the temperature distribution for the Venus disk at $230 \mathrm{~K}$ can be estimated at $1.26 \mathrm{~K}$. This result meets the design specification before the launch.

\section{Discussion}

Brightness temperature of the Venus disk.

Figure 7 shows the same as Fig. 6 except that the brightness temperature distribution is for the Venus disk. A thermal infrared image of Venus taken by Pioneer Venus orbiter (PVO) showed the limb darkening effect in which temperature distribution systematically decreases as a function of the zenith angle of the emission (Taylor 1980). The effect depended on the vertical structure of the Venus atmosphere, the local time, and the emission angle at the time of observation. Images of the Venus disk obtained by LIR also exhibit the limb darkening effect 

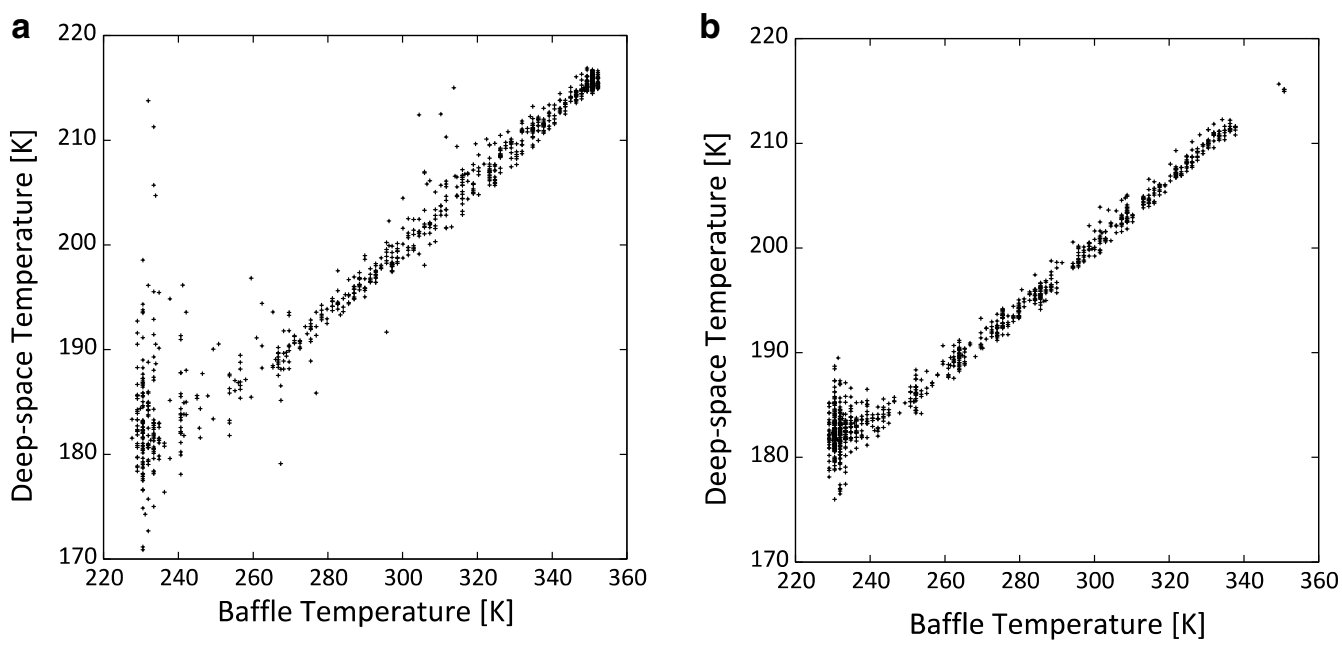

Fig. 3 Distribution of the brightness temperature retrieved from the deep-space area in LIR images with $m=32$ and $n=32$ as a function of the baffle temperature. a Data acquired from March to September 2016 with LIR turned on 15 min before the beginning of each observation. b Data acquired from October to December 2016 with the power supply unit of LIR continuously powered on (since in October 2016)

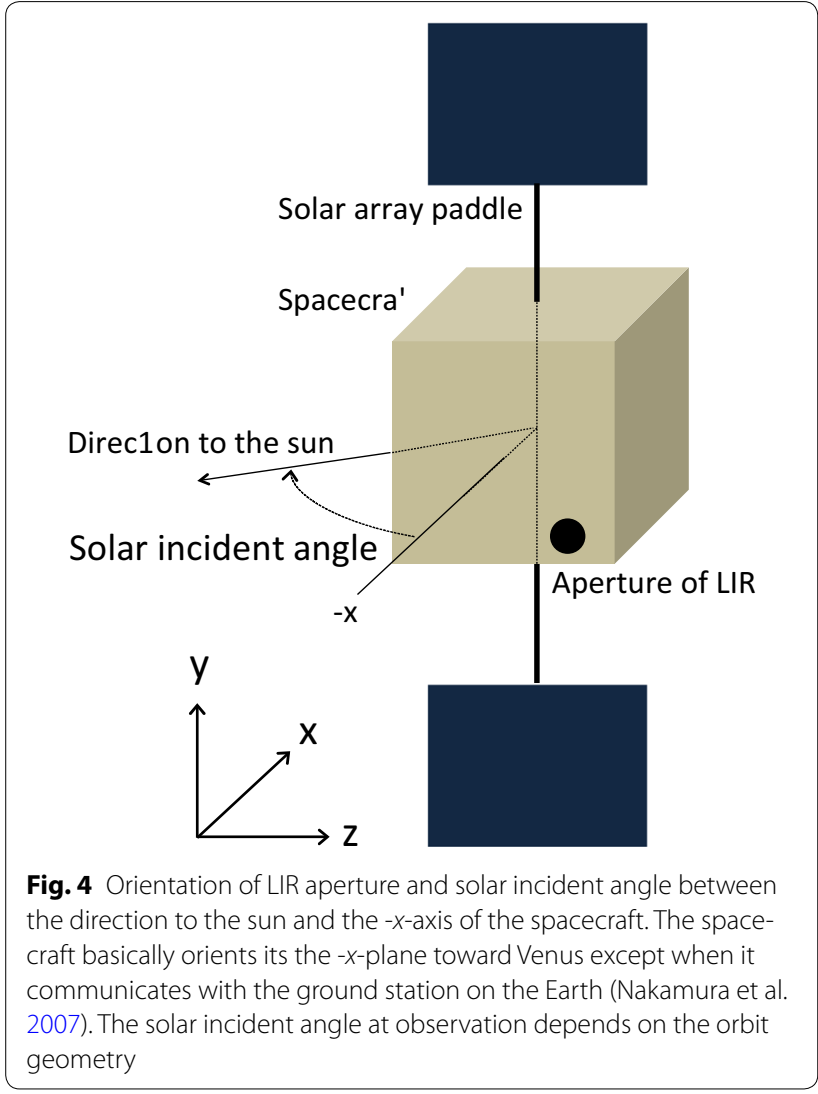

even after correction of the background bias. The effect should be carefully removed with consideration of the atmospheric conditions. The effect is negligible at the disk center, where the emission angle is low. Therefore, the mean brightness temperature on the Venus disk after the correction in Fig. 7 was simply obtained within the area of an emission angle of less than $10^{\circ}$; they mostly converged to $\sim 230 \mathrm{~K}$ as expected without dependence on the baffle temperature. The result is consistent with the temperature deduced from previous studies, as described in Introduction. On the other hand, the brightness temperature derived in Taguchi et al. (2012) is obviously inconsistent with this result. However, we were not aware of the background bias in 2010; the baffle temperature ranged up to $327.4 \mathrm{~K}$ at the time of observation. When we applied the correction of the background bias to the Venus disk image observed in 2010, the brightness temperature predictably decreased to $\sim 231 \mathrm{~K}$.

\section{Origin of the background bias}

Since infrared radiation from the baffle does not directly enter the UMBA, we assumed at the time of the design process that the image brightness was completely independent of the baffle temperature. However, the observed bias suggests that the baffle has radiative coupling with the Ge lens, and the radiative coupling may indirectly affect image brightness when the baffle is heated. The baffle was cooled to $230 \mathrm{~K}$ in the laboratory experiment before the launch; however, the baffle heating by solar radiation could not be reproduced in this experiment. The temperature of the Ge lens is monitored via telemetry and kept thermally stable by a heater to prevent radiative coupling. However, the thermal sensors are not put on the lens itself but on the lens holder, as shown in Fig. 8. Thus, the thermal sensor may not measure the actual temperature of the Ge lens when the baffle is temporarily heated. 
a

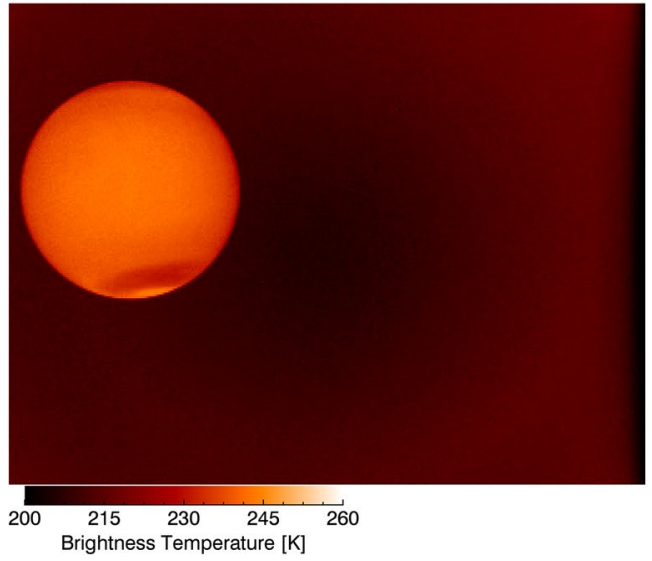

b

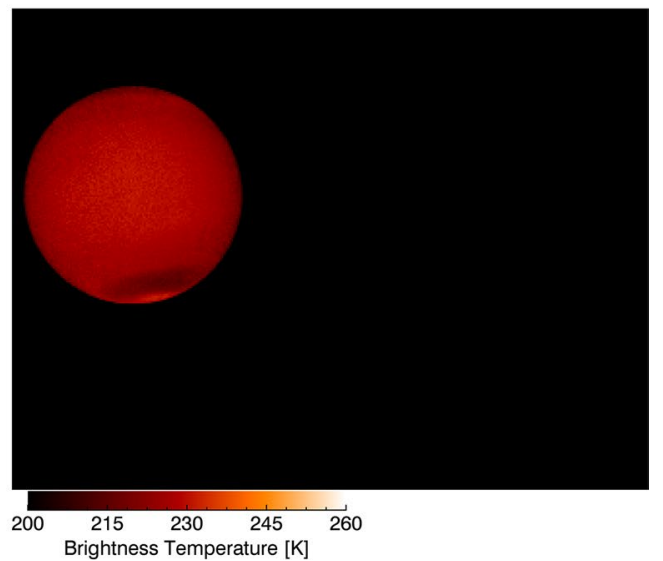

Fig. 5 a Original LIR image representing the brightness temperature of the Venus disk. It was acquired on October 19, 2016, with the baffle temperature being 334.6 K. b The same image corrected for the background bias

The temperature difference between the lens and the lens holder was verified in the heating scenario by using the proto model of LIR in an air environment in the laboratory. In order to measure the actual lens temperature, thermocouples were put directly on the center of the Ge lens and the lens holder where temperature for the telemetry is measured. A sheet heater was placed in front of the lens in Fig. 8 to simulate infrared radiation from the baffle, and it was heated from 300 to $320 \mathrm{~K}$. When the temperature of the heater attained a maximum of $320 \mathrm{~K}$, the temperature of the Ge lens, which was initially equivalent to that of the lens holder,

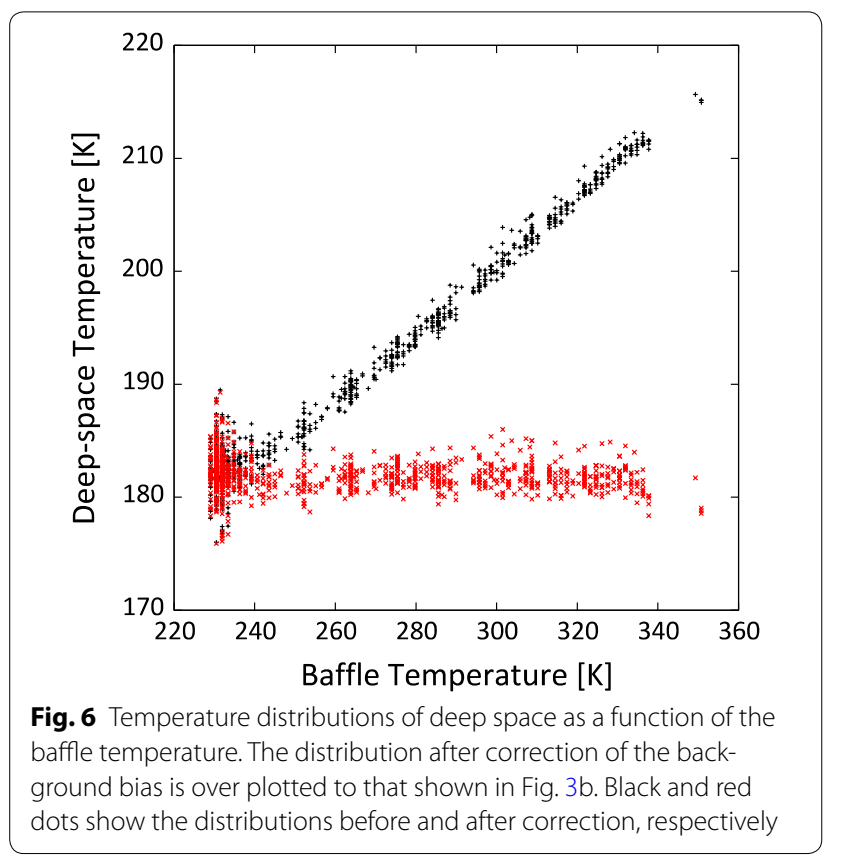

became $\sim 0.6 \mathrm{~K}$ higher than that of the lens holder. Temperature of the Ge lens on orbit also must become higher than that of the lens holder monitored by the thermal sensor when the baffle is temporarily heated by solar radiation. Thus, we confirmed that the temperature difference between the Ge lens and the lens holder probably caused the background bias seen in the LIR images.

\section{Conclusions}

The result of initial checkouts on orbit indicated that LIR worked without serious faults despite 5 years of extra cruise under harsh solar exposure. However, early LIR images contained a noticeable background bias dependent on the thermal condition of the instrument. When the power supply unit for LIR was kept active since October 2016, the bias could be partially removed from the images. The residual component was simply correlated with the baffle temperature and was successfully corrected by using a reference table based on the deep-space images acquired at different baffle temperatures on orbit. The cloud-top temperature at the center of the Venus disk retrieved from LIR observation was consistent with that deduced from previous studies. The reference table for the correction is provisional; an authentic reference table will be shortly provided based on the deep-space images acquired with the thermal behavior of the baffle strictly controlled.

An observation program with $m=32$ and $n=1$ was additionally prepared for the acquisition of the close-up images with a high spatial resolution. This observation program enables us to investigate not only the fine structure of the cloud-top temperature but also the horizontal temperature distribution along an entire longitude 


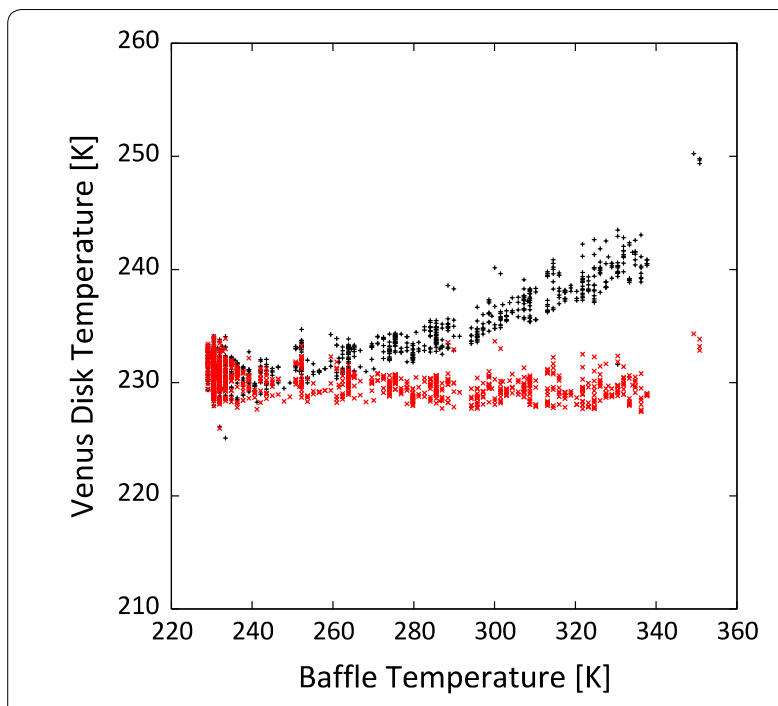

Fig. 7 Same as Fig. 6 but for temperature distributions at the center of the Venus disk

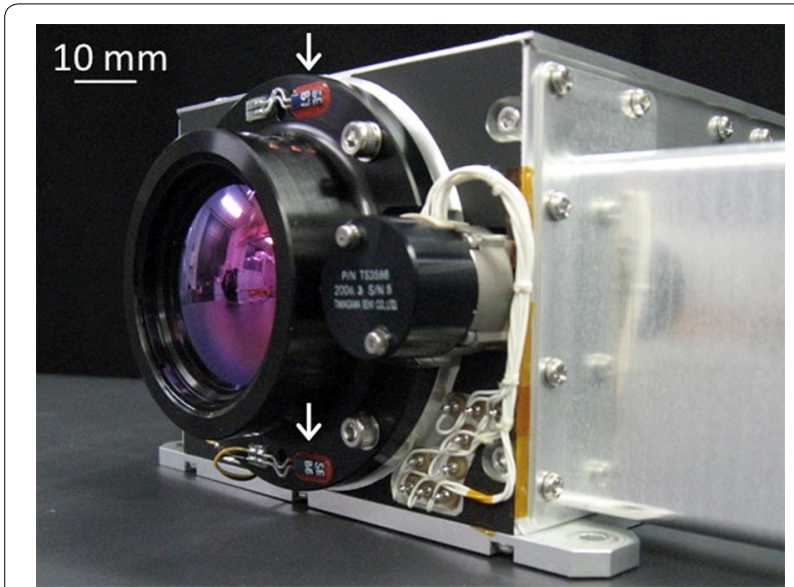

Fig. 8 Photograph of the Ge lens mounted to the LIR aperture. White arrows indicate positions of the thermal sensors put on the lens holder

during orbit. When the whole longitude is continuously observed, the local time dependence of the cloud-top temperature will be revealed.

We must note that the behavior of the baffle temperature during periapsis passage is not completely understood because the solar incident angle varies rapidly there. We have attempted to eliminate the background bias from the images acquired near periapsis using formula (4); however, the deep-space temperature still contained a residual component of the background bias. Thus, the background bias in the images acquired near periapsis currently cannot be corrected by the procedure described in this paper. When more Venus images are acquired near periapsis, fluctuation of the baffle temperature will be systematically revealed enough to eliminate the residual background bias.

\section{Authors' contributions}

MT is the principal investigators of $L I R$. TF, MF, GLH, TI, NI, TK, MN, MS, TMS, MS, $S T, M U, A H, T Y$, and $M T$ are co-investigators of LIR. AY, MY, SM, YY, KO, HA, KS, $\mathrm{HK}, \mathrm{SO}, \mathrm{NI}, \mathrm{TA}, \mathrm{TS}, \mathrm{CH}$, and $\mathrm{NH}$ contribute to operation of Akatsuki and image processing of LIR. All authors read and approved the final manuscript.

\section{Author details}

${ }^{1}$ College of Science, Rikkyo University, 3-34-1 Nishi-lkebukuro, Toshima-ku, Tokyo 171-8501, Japan. ${ }^{2}$ Graduate School of Frontier Sciences, The University of Tokyo, Kiban-tou 4H7, 5-1-5 Kashiwanoha, Kashiwa, Chiba 277-8561, Japan. ${ }^{3}$ Omori Medical Center, Toho University, 6-11-1 Omorinishi, Ota-ku, Tokyo 143-8541, Japan. ${ }^{4}$ National Institute of Advanced Industrial Science and Technology, 2-3-26 Aomi, Koto-ku, Tokyo 135-0064, Japan. ${ }^{5}$ Institute of Space and Astronautical Science, Japan Aerospace Exploration Agency, 3-1-1, Yoshinodai, Chuo-ku, Sagamihara, Kanagawa 252-5210, Japan. ${ }^{6}$ Senshu University, 2-1-1 Higashimita, Tama-ku, Kawasaki, Kanagawa 214-8580, Japan. ${ }^{7}$ Department of Planetology, Center for Planetary Science, Kobe University, 7-1-48, Minatojima-Minamimachi, Chuo-ku, Kobe 650-0047, Japan. ${ }^{8}$ Department of Earth Sciences, Okayama University, 3-1-1 Tsushimanaka, Kita-ku, Okayama 700-8530, Japan. ${ }^{9}$ Faculty of Environmental Earth Science, Hokkaido University, N10W5, Sapporo, Hokkaido 060-0810, Japan. ${ }^{10}$ Research and Information Center, Tokai University, 4-1-1 Kitakaname, Hiratsuka-shi, Kanagawa 259-1292, Japan. ${ }^{11}$ Chiba Institute of Technology, Narashino, Chiba, Japan. ${ }^{12}$ The University of Shiga Prefecture, 2500, Hassaka-cho, Hikone-City, Shiga 522-8533, Japan. ${ }^{13}$ Faculty of Science, Kyoto Sangyo University, Motoyama, Kamigamo, Kita-ku, Kyoto 603-8555, Japan. ${ }^{14}$ Matsue College, National Institute of Technology, 14-4 Nishiikuma-cho, Matsue, Shimane 690-8518, Japan.

${ }^{15}$ The University of Aizu, Aizu-Wakamatsu, Fukushima Prefecture 965-8580, Japan.

\section{Competing interests}

The authors declare that they have no competing interests.

\section{Publisher's Note}

Springer Nature remains neutral with regard to jurisdictional claims in published maps and institutional affiliations.

Received: 26 July 2017 Accepted: 29 September 2017

Published online: 06 October 2017

\section{References}

Fukuhara T et al (2011) LIR: Longwave infrared camera onboard the Venus orbiter Akatsuki. Earth Planets Space 63:1009-1018. doi:10.5047/ eps.2011.06.019

Fukuhara T et al (2017) Large stationary gravity wave in the atmosphere of Venus. Nat Geosci. doi:10.1038/NGEO2873

Imamura T et al (2011) RS: Radio Science investigation of the Venus atmosphere and ionosphere with Venus orbiter, Akatsuki. Earth Planets Space 63:493-501. doi:10.5047/eps.2011.03.009

Iwagami $\mathrm{N}$ et al (2011) Science requirements and description of the $1 \mu \mathrm{m}$ camera onboard the Akatsuki Venus Orbiter. Earth Planets Space 63:487-492. doi:10.5047/eps.2011.03.007

Nakamura M et al (2007) Planet-C: Venus climate orbiter mission of Japan. Planet Space Sci 55:1831-1842

Nakamura M et al (2011) Overview of Venus orbiter, Akatsuki. Earth Planets Space 63:443-457. doi:10.5047/eps.2011.02.009

Nakamura M et al (2016) AKATSUKI returns to Venus. Earth Planets Space 68:75. doi:10.1186/s40623-016-0457-6

Pollack JB et al (1993) Near-infrared light from Venus' nightside: a spectroscopic analysis. Icarus 103:1-42

Satoh T et al (2015) Venus'clouds as Inferred from the phase curves acquired by IR1 and IR2 onboard Akatsuki. Icarus 248:213-220 
Seiff A et al (1985) Models of the structure of the atmosphere of Venus from the surface to 100 kilometers altitude. Adv Space Res 5(11):3-58

Taguchi M et al (2007) Longwave infrared camera onboard the Venus climate orbiter. Adv Space Res 40:861-868

Taguchi M et al (2012) Characteristic features in Venus' nightside cloud-top temperature obtained by Akatsuki/LIR. Icarus 219:502-504

Taylor FW (1980) Structure and meteorology of the middle atmosphere of

Venus: infrared remote sensing from the pioneer orbiter. J Geophys Res 85(A13):7963-8006
Tellman S (2009) Structure of the Venus neutral atmosphere as observed by the Radio Science experiment VeRa on Venus Express. J Geophys Res 114:E00B36. doi:10.1029/2008JE003204

Zasova LV et al (2007) Structure of the Venus atmosphere. Planet Space Sci $55: 1712-1728$

\section{Submit your manuscript to a SpringerOpen ${ }^{\odot}$} journal and benefit from:

- Convenient online submission

- Rigorous peer review

- Open access: articles freely available online

- High visibility within the field

- Retaining the copyright to your article

Submit your next manuscript at $\boldsymbol{\nabla}$ springeropen.com 